CORRECTION

\title{
Correction to: Globalization and global governance
}

\section{Zheng Chen ${ }^{1}$}

Published online: 28 January 2020

(c) The Institute of International and Strategic Studies (IISS), Peking University 2020

\section{Correction to: China International Strategy Review (2019) https://doi.org/10.1007/s42533-019-00020-z}

The article title was incorrect. The correct title is "Globalization and global governance".

The original article can be found online at https://doi.org/10.1007/s42533-019-00020-z.

\section{Zheng Chen} chenzhengsjtu@foxmail.com

1 School of International and Public Affairs, Shanghai Jiao Tong University, Shanghai, China 